\title{
SMOOTH EXTENSIONS FOR A FINITE CW COMPLEX
}

\author{
GUIHUA GONG
}

The $C^{*}$-algebra extensions of a topological space can be made into an abelian group which is naturally equivalent to the $K$ homology group of odd dimension [1] which has a close relation with index theory and is one of the starting points of $K K$ theory [8].

The $C_{p}$-smoothness of an extension of a manifold was introduced in [3, 4], where $C_{p}$ denotes the Schatten-von Neumann $p$-class [5]. We generalize the notion of $C_{p}$-smoothness to a finite $\mathrm{CW}$ complex and obtain necessary and sufficient conditions for an extension of a finite CW complex to be $C_{p}$-smooth modulo torsion.

The notion of $C_{p}$-smooth extensions is one of the motivations for Connes' cyclic cohomology. In [2] Connes constructs a Chern map from $K K(C(M), \mathrm{C})$ to the cyclic cohomology of $C^{\infty}(M)$, and proves that this Chern map is a surjection modulo torsion. One consequence of the even counterpart of our main results is that this Chern map is a graded surjection modulo torsion. We will make this statement precise in Theorem 3.

Let $H$ be an infinite dimensional complex separable Hilbert space. By $L(H)$ and $K(H)$ we shall denote the $C^{*}$-algebra of bounded operators and compact operators on $H$, respectively, and $Q(H)$ will denote the quotient $L(H) / K(H)$ with canonical surjection $\pi: L(H) \rightarrow Q(H)$. For $X$ a compact metrizable space an extension $\tau \in \operatorname{Ext}(X)$ of the algebra $C(X)$ by $K(H)$ is defined by a unital $*$ monomorphism $\tau: C(X) \rightarrow Q(H)[1]$.

Definition 1. Let $M$ be a smooth compact manifold (perhaps with boundary) and let $C^{\infty}(M)$ denote the $*$-algebra of all smooth functions on $M$. A $\tau \in \operatorname{Ext}(M)$ is $C_{p}$-smooth if there exists a *linear map $\rho: C^{\infty}(M) \rightarrow L(H)$ such that $\rho(a b)-\rho(a) \rho(b) \in C_{p}$ and $\pi \circ \rho=\tau \mid C^{\infty}(M)$.

\footnotetext{
Received by the editors October 15, 1988 and, in revised form, July 10, 1989. $46 \mathrm{~L} 80$.

1980 Mathematics Subject Classification (1985 Revision). Primary 19K33,

The author is partially supported as graduate assistant on a grant from the NSF. 
This definition can be found in [2] and is equivalent to the definition in [4] by means of the $C^{\infty}$ functional calculus of HeltonHowe $[6,7]$.

In order to define $C_{p}$-smooth for a general finite $\mathrm{CW}$ complex, we shall use the following Lemma:

Lemma. If $X$ is a finite $\mathrm{CW}$ complex, then there exist a compact smooth manifold $M$ (perhaps with boundary), and two maps $f: X \rightarrow M$ and $g: M \rightarrow X$ such that $(g \circ f)$ is homotopic to id $\mid X$.

Definition 2. Let $X, M$ and $f$ be as in the Lemma. Then $\tau \in$ $\operatorname{Ext}(X)$ is $C_{p}$-smooth if $f_{*} \tau \in \operatorname{Ext}(M)$ is $C_{p}$-smooth.

It is not difficult to prove that the $C_{p}$-smoothness does not depend on the choice of $M$ and the maps by using the following fact: Any continuous map between two smooth manifolds is homotopic to a smooth map. Similarly, we prove that the notion of $C_{p}$-smoothness of a manifold does not depend on the particular differential structure which answers the question on p. 68 of [3]. And also we prove that if $f: X \rightarrow Y$ is a continuous map between finite CW complexes $X$ and $Y$, then $f_{*}$ maps the $C_{p}$-smooth elements of $\operatorname{Ext}(X)$ to the $C_{p}$-smooth elements of $\operatorname{Ext}(Y)$.

Our main results are Theorems 1, 2, 3 .

Theorem 1. Let $X$ be a finite $\mathrm{CW}$ complex, $X^{k}$ denote the $k$ skeleton of $X$, and $\tau \in \operatorname{Ext}(X)$. Then there exists an integer $m_{1} \neq 0$ such that $m_{1} \tau$ is $C_{n}$-smooth if and only if there exists an integer $m_{2} \neq 0$ such that $m_{2} \tau \in i_{*}\left(\operatorname{Ext}\left(X^{2 n-1}\right)\right)$, where $i_{*}: \operatorname{Ext}\left(X^{2 n-1}\right) \rightarrow \operatorname{Ext}(X)$ is induced by the inclusion map $i:$ $X^{2 n-1} \rightarrow X$. Furthermore, if $X$ is a smooth compact $(2 n-1)$ manifold, then each element in $\operatorname{Ext}(X)$ is $C_{p}$-smooth when $p>$ $n-\frac{1}{2}$.

The "only if" part of Theorem 1 generalizes the results in [3, 4]. It was shown in $[3,6]$ that the $C_{1}$-smooth elements of $\operatorname{Ext}(X)$ come from the 1-skeleton modulo torsion. And also it was shown in [4] that each $C_{n-1}$-smooth element of $\operatorname{Ext}\left(S^{2 n-1}\right)$ is trivial.

As a corollary of Theorem 1, we know that all the elements of $\operatorname{Ext}\left(S^{2 n-1}\right)$ are $C_{p}$-smooth when $p>n-\frac{1}{2}$. This result solves the problem on p. 109 of [4]. As a special case, we have the following fact: If $\left(T_{z_{1}}, T_{z_{2}}, \ldots, T_{z_{n}}\right)$ is the $n$-tuple of Toeplitz operators on $H^{2}\left(\partial B_{n}\right)$, then there exist $n$ compact operators $K_{1}, K_{2}, \ldots, K_{n}$ such that $\left[T_{z_{i}}+K_{i}, T_{z_{j}}+K_{j}\right] \in C_{p}$ and $\left[T_{z_{i}}+K_{i}, T_{z_{j}}^{*}+K_{j}^{*}\right] \in C_{p}$ 
when $p>n-\frac{1}{2}$. There doesn't seem to be any direct proof of this. The author does not know whether the elements of $\operatorname{Ext}\left(S^{2 n-1}\right)$ are $C_{p}$-smooth when $n-1<p \leq n-\frac{1}{2}$.

The following result is almost equivalent to Theorem 1 but is perhaps more useful in practice.

Theorem 2. Let $X$ be a finite CW complex, $\tau \in \operatorname{Ext}(X)=K_{1}(X)$ and ch: $K_{1}(X) \otimes \mathbf{C} \rightarrow H_{\text {odd }}(X, \mathbf{C})$ be the Chern map, where $H_{\text {odd }}(X, \mathbf{C})$ denotes the direct sum of all the ordinary homology groups with complex coefficients of odd dimension. Then there exists an integer $m \neq 0$ such that $m \tau$ is $C_{n}$-smooth if and only if $\operatorname{ch} \tau \in \sum_{k=1}^{n} H_{2 k-1}(X, \mathbf{C})$.

We also obtain some similar results about the $p$-summable Fredholm modules of $C^{\infty}(M)$, which can be thought of as elements of $K_{0}(M)=K K(C(M), C)$, and about their Chern characters in the cyclic cohomology $H_{\lambda}^{*}\left(C^{\infty}(M)\right)$. In particular, we prove the following theorem.

Theorem 3. If $M$ is a compact smooth manifold without boundary and $\varphi \in H_{\lambda}^{k}\left(C^{\infty}(M)\right)$ ( $k$ even $)$, then there exist $(k+1)$ summable Fredholm modules $\tau_{i}(i=1,2, \ldots, n)$ and complex numbers $\alpha_{i}(i=1,2, \ldots, n)$ such that $\sum_{i=1}^{n} \alpha_{i} \operatorname{ch}^{*} \tau_{i} \sim \varphi$ in $H_{\lambda}^{*}\left(C^{\infty}(M)\right)$, where $\mathrm{ch}^{*}$ is Connes' Chern map.

We would like to point out that A. Connes constructed the graded Chern characters

$\operatorname{ch}^{*}:\{n+1$ summable Fredholm module $\} \rightarrow H_{\lambda}^{n}\left(C^{\infty}(M)\right)$

in $\S 2$ of [2], and that he also proved that

$\mathrm{ch}^{*}:\{$ finite summable Fredholm module $\} \rightarrow H_{\lambda}^{*}\left(C^{\infty}(M)\right)$

is surjective modulo torsion. Theorem 3 says that the

Chern map is a graded surjection.

In order to prove our main theorems, we need some results from topology. Theorem 5 is a special case of the theorem on p. 210 line 7 of [9]. And Theorem 4 is perhaps also familiar to topologists. We provide an outline of a proof for Theorem 4 since we have been unable to find a precise reference.

Theorem 4. Let $X$ be compact metrizable space. For any $\tau \in$ $K^{1}(X)$, there exist maps $f_{i}: X \rightarrow S^{2 n_{i}-1}(i=1,2, \ldots, k)$ such that $m \tau=\sum_{i=1}^{k} f_{i}^{*} \theta_{i}$ for some integer $m$, where $\theta_{i}$ is the canonical generator of $K^{1}\left(S^{2 n_{i}-1}\right)$.

Theorem 5. If $X$ is a finite $\mathrm{CW}$ complex and $\tau \in H_{k}(X)$, then there exist a smooth compact oriented $k$-manifold $M$ without 
boundary and a map $f: M \rightarrow X$ such that $m \tau=f_{*} \theta$ for some integer $m \neq 0$ and $\theta \in H_{k}(M)$.

To prove Theorem 4, we only need to prove the case of $X=$ $U(n)$ because each element of $K^{1}(X)$ can be realized as the pullback of an element in $K^{1}(U(n))$ via a map from $X$ to $U(n)$. The idea is to use obstruction theory and a result about Whitehead products [10, Theorem 8.9] to construct two maps: $u: S^{1} \times$ $S^{3} \times \cdots \times S^{2 n-1} \rightarrow U(n), v: U(n) \rightarrow S^{1} \times S^{3} \times \cdots \times S^{2 n-1}$, such that

$$
\begin{aligned}
& (v \circ u)^{*}: K^{1}\left(S^{1} \times S^{3} \times \cdots \times S^{2 n-1}\right) \\
& \quad=Z^{2^{n-1}} \rightarrow K^{1}\left(S^{1} \times S^{3} \times \cdots \times S^{2 n-1}\right)
\end{aligned}
$$

can be represented by a matrix

$$
\left(\begin{array}{cccc}
m_{1} & 0 & \cdots & 0 \\
0 & m_{2} & \cdots & 0 \\
& \cdots & \cdots & 0 \\
0 & 0 & \cdots & m_{2^{n-1}}
\end{array}\right)
$$

where $m_{k} \neq 0$ are integers, and $(u \circ v)^{*}: K^{1}(U(n))=Z^{2^{n-1}} \rightarrow$ $K^{1}(U(n))$ has the same form as $(v \circ u)^{*}$. Then we can reduce the problem to the case of $S^{1} \times S^{3} \times \cdots \times S^{2 n-1}$ which can be easily done.

Using Proposition 3 in [4] and Theorem 4, we can prove the "only if" part of Theorem 1. For the "if" part we use Theorem 5.

The even counterpart of Theorems 1, 2 can be obtained in a similar manner and this is used in proving Theorem 3.

\section{ACKNOWLEDGMENTS}

The author wishes to thank Professor Ronald G. Douglas for suggesting the topic of this paper and many helpful discussions. Thanks also go to Professors Nicholae Teleman and Lowell Jones for topological consultations.

\section{REFERENCES}

1. L. G. Brown, R. G. Douglas, and P. A. Fillmore, Extensions of $C^{*}$-algebra and K-homology, Ann. of Math. (2) 165 (1977), 265-324.

2. A. Connes, Non commutative differential geometry, Chapters 1,2, Publ. Math. Inst. Hautes Études Sci. 62 (1986), 257-360.

3. R. G. Douglas, On the smoothness of elements of Ext, Topics in Modern Operator Theory, Birkhauser-Verlag, 1981, pp. 63-69.

4. R. G. Douglas and D. Voiculescu, On the smoothness of sphere extensions, J. Operator Theory 6 (1981), 103-111. 
5. I. C. Gohberg and M. G. Krein, Introduction to the theory of linear nonselfadjoint operators, vol. 18, Transl. Math. Monographs, Amer. Math. Soc., Providence, R.I., 1969.

6. J. W. Helton and R. Howe, Integral operators: commutators, traces, index and homology, Proc. Conf. on Operator Theory, Lecture Notes in Math., vol 345, Springer-Verlag, Berlin and New York, 1973, pp. 141-209.

7. _ Traces of commutators of integral operators, Acta Math. 135 (1975), 271-305.

8. G. Kasparov, The operator $K$-functor and extensions of $C^{*}$-algebras, Izv. Akad. Nauk SSSR Ser. Math. 44 (1980), 571-636; Math. USSR Izvestija 16 (1981), 513-572.

9. R. E. Stong, Notes on cobordism theory, Chapter IX, Mathematical Notes, Princeton Univ. Press, Princeton, N.J., 1968.

10. G. W. Whitehead, Elements of homotopy theory, Chapter 11, Springer-Verlag, 1978.

Department of Mathematics, State University of New York at Stony Brook, Stony Brook, New York 11794 
